\title{
Supporting Information for Iron Substitution for Sodium in a Carboxylate-Bridged, Heterodinuclear Sodium-Iron Complex
}

\author{
Jeremy J. Kodanko, Dong Xu, Datong Song and Stephen J. Lippard* \\ Department of Chemistry, Massachusetts Institute of Technology, Cambridge, \\ Massachusetts, 02139
}

\section{Experimental Section}

General Considerations. All reagents were obtained from commercial sources and used as received unless otherwise noted. Acetonitrile $(\mathrm{MeCN})$, diethyl ether $\left(\mathrm{Et}_{2} \mathrm{O}\right)$ and dichloromethane $\left(\mathrm{CH}_{2} \mathrm{Cl}_{2}\right)$ were saturated with nitrogen and purified by passing though activated alumina columns under argon. ${ }^{1}$ 1,2-Dichloroethane (DCE) and chlorobenzene $(\mathrm{ClPh})$ were obtained in Aldrich Sure-Seal ${ }^{\circledR}$ bottles and were deoxygenated by bubbling Ar through a submerged needle for $1 \mathrm{~h}$ before use. All reactions and manipulations were performed in an Mbraun drybox under a nitrogen atmosphere. Synthesis of the dipicolinic ester ligand $\mathrm{PIC}_{2} \mathrm{DET}$ (1) was carried out as described elsewhere. ${ }^{2}$ The sodium salt $\mathrm{NaO}_{2} \mathrm{CTrp}$, where Trp is 9-triptycenyl, was obtained by reaction of $\mathrm{HO}_{2} \mathrm{CTrp}^{3}$ with $\mathrm{NaOH}$ in $\mathrm{MeOH}$ by a standard method. ${ }^{4}$

[NaFe(PIC ${ }_{2}$ DET $)\left(\mu-O_{2} \text { CTrp) }\right)_{3}$ (2). A mixture of $\mathrm{NaO}_{2}$ CTrp (168 mg, 525 $\mu \mathrm{mol}), \mathrm{Fe}(\mathrm{OTf})_{2} \cdot 2 \mathrm{MeCN}(76 \mathrm{mg}, 175 \mu \mathrm{mol}), \mathrm{PIC}_{2} \mathrm{DET}$ (1) $(100 \mathrm{mg}, 175 \mu \mathrm{mol})$ and DCE $(5 \mathrm{~mL})$ was stirred for $1 \mathrm{~h}$ under a nitrogen atmosphere, resulting in a blue-green heterogeneous mixture. The colorless solid $\mathrm{NaOTf}^{5}$ was removed by filtration, and the blue-green filtrate was concentrated under reduced pressure, leaving a purple solid. Vapor diffusion of $\mathrm{Et}_{2} \mathrm{O}$ into a $\mathrm{ClPh}$ solution of the purple solid gave blue blocks of the complex $2 \cdot 2 \mathrm{ClPh}(222 \mathrm{mg}, 126 \mu \mathrm{mol}, 71 \%)$ that were isolated by filtration after 2 days. 
Crystals of 2 suitable for X-ray analysis were obtained by diffusion of $\mathrm{Et}_{2} \mathrm{O}$ into a $\mathrm{CH}_{2} \mathrm{Cl}_{2}$ solution of the compound. A mixture of crystalline materials was obtained by this method, consisting mainly of blue-yellow dichroic blocks, with a small amount $(<5 \%)$ of purple-colorless dichroic blocks. X-ray crystallographic data for the blue-yellow blocks (Structure A) and purple-colorless blocks (Structure B) are provided below. IR $\left(\mathrm{KBr}, \mathrm{cm}^{-1}\right) 3062(\mathrm{~m}), 2952(\mathrm{~m}), 2856(\mathrm{w}), 2203(\mathrm{w}), 1723(\mathrm{~m}), 1691(\mathrm{w}), 1624(\mathrm{~s}), 1582$ (m), 1561 (w), 1476 (w), 1458 (s), 1444 (s), 1382 (s), 1340 (m), 1319 (s), 1287 (m), 1239 (m), $1199(w), 1176(w), 1151(w), 1114(w), 1078(w), 1031(w), 874(w), 794(w), 749$ (s), 723 (w), 698 (w), 687 (w), 627 (s). The UV-vis spectra of 2 in $\mathrm{CH}_{2} \mathrm{Cl}_{2}$ and $\mathrm{CH}_{3} \mathrm{CN}$ are displayed in Figs. S1 and S2, respectively. Anal. Calcd. for $\mathrm{C}_{101} \mathrm{H}_{63} \mathrm{FeN}_{2} \mathrm{NaO}_{10} \cdot 2 \mathrm{C}_{6} \mathrm{H}_{5} \mathrm{Cl}: \mathrm{C}, 76.74 ; \mathrm{H}, 4.16 ; \mathrm{N}, 1.58$. Found: C, 76.65; H, 4.29; N, 1.72. Metal analyses were performed on a sample that had been dried in vacuo at $50{ }^{\circ} \mathrm{C}$. Anal. Calcd. for $\mathrm{C}_{101} \mathrm{H}_{63} \mathrm{FeN}_{2} \mathrm{NaO}_{10}$ : $\mathrm{Fe}, 3.62 ; \mathrm{Na}, 1.49$. Found: $\mathrm{Fe}, 3.61 ; \mathrm{Na}, 1.98$.

$\left[\mathrm{Fe}_{2}\left(\mathbf{P I C}_{2} \mathbf{D E T}\right)\left(\mu-\mathrm{O}_{2} \mathbf{C T r p}\right)_{3}\right][\mathbf{O T f}](3)$. A mixture of 2-2ClPh (50 mg, $\left.28 \mu \mathrm{mol}\right)$, $\mathrm{Fe}(\mathrm{OTf})_{2} \cdot 2 \mathrm{MeCN}(70 \mathrm{mg}, 160 \mu \mathrm{mol})$ and DCE $(2 \mathrm{~mL})$ was stirred for $45 \mathrm{~min}$, resulting in a color change from blue to red. The reaction mixture was filtered and the filtrate was concentrated under reduced pressure to give $\left[\mathrm{Fe}_{2}(\mathbf{1})\left(\mu-\mathrm{O}_{2} \mathrm{CTrp}\right)_{3}\right][\mathrm{OTf}](\mathbf{3})(49 \mathrm{mg}, 28$ $\mu \mathrm{mol}, 100 \%)$ as an air-sensitive red solid. Vapor diffusion of $\mathrm{Et}_{2} \mathrm{O}$ into a $\mathrm{MeCN}$ solution of the red solid gave a microcrystalline purple solid that was not amenable to X-ray analysis. A similar reaction was performed in $\mathrm{CH}_{2} \mathrm{Cl}_{2}$ and was followed by $\mathrm{UV}$-vis spectroscopy (Fig. S1). IR (KBr, cm $\left.{ }^{-1}\right) 3060$ (m), 2957 (m), 2924 (w), 2847 (w), 2207 (w), $1684(\mathrm{w}), 1614(\mathrm{~s}), 1564(\mathrm{~m}), 1487(\mathrm{w}), 1459(\mathrm{~s}), 1445(\mathrm{~s}), 1410(\mathrm{~m}), 1350(\mathrm{~s}), 1314$ (m), $1292(\mathrm{~m}), 1236(\mathrm{~s}), 1212(\mathrm{~s}), 1177(\mathrm{~m}), 1031(\mathrm{~s}), 750(\mathrm{~s}), 721(\mathrm{w}), 696(\mathrm{w}), 647(\mathrm{w})$, 
637 (m), 626 (s). The UV-vis spectra of 3 in $\mathrm{CH}_{2} \mathrm{Cl}_{2}$ and $\mathrm{CH}_{3} \mathrm{CN}$ are displayed in Figs. S1 and S2, respectively. LRMS (ESMS) Calcd for $\mathrm{C}_{101} \mathrm{H}_{63} \mathrm{Fe}_{2} \mathrm{~N}_{2} \mathrm{O}_{10}$ (M-OTf) ${ }^{+}: 1576$, Found: 1576.

UV-vis Titration Experiment. In a $5.00 \mathrm{~mL}$ cuvette fitted with a rubber septum, a solution of $2 \cdot 2 \mathrm{ClPh}(8.9 \mathrm{mg}, 5.0 \mu \mathrm{mol})$ and $\mathrm{MeCN}(5.00 \mathrm{~mL})$ was treated with a solution of $\mathrm{Fe}(\mathrm{OTf})_{2} \cdot 2 \mathrm{MeCN}(8.7 \mathrm{mg}, 20 \mu \mathrm{mol})$ and $\mathrm{MeCN}(200 \mu \mathrm{L})$ in $25 \mu \mathrm{L}$ portions. After each portion was added, the reaction was shaken until the solution was homogenous, and then the UV-vis spectrum was acquired (Fig. S3).

X-ray Crystallographic Studies. Intensity data were collected on a Bruker (formerly Siemens) SMART APEX CCD diffractometer with graphite-monochromated Mo K $\alpha$ radiation $(\lambda=0.71073 \AA$ ), controlled by a Pentium-based PC running the SMART software package. ${ }^{6}$ Single crystals were mounted on the tips of glass fibers, coated with paratone- $\mathrm{N}$ oil, and cooled to $173 \mathrm{~K}$ under a stream of $\mathrm{N}_{2}$ maintained by a KRYO-FLEX low-temperature apparatus. Data collection and reduction protocols are described elsewhere. ${ }^{7}$ The structures were solved by both direct and Patterson methods and refined on $\mathrm{F}^{2}$ by using the SHELXTL-97 software $^{8}$ incorporated in the SHELXTL software package. ${ }^{9}$ Empirical absorption corrections were applied by using the SADABS program, ${ }^{10}$ and the structures were checked for higher symmetry with the PLATON software. ${ }^{11}$ All non-hydrogen atoms were located and their positions refined with anisotropic thermal parameters by least-squares cycles and Fourier syntheses. All hydrogen atoms were assigned to idealized positions and given thermal parameters equivalent to either 1.5 (methyl hydrogen atoms) or 1.2 (all other hydrogen atoms) times the thermal parameter of the carbon atom to which they were attached. In Structure A 
there is a disordered $\mathrm{Et}_{2} \mathrm{O}$ solvent molecule that was refined at $50 \%$ occupancy. The DFIX constraint was used to set the $\mathrm{C}-\mathrm{C}$ and $\mathrm{C}-\mathrm{O}$ bond distances on this $\mathrm{Et}_{2} \mathrm{O}$ solvent molecule. The molecular structure and a view highlighting the coordination geometry are depicted in Figs. S4 and S5, respectively. In structure B, four disordered dichloromethane solvent molecules were removed using the Squeeze function of the PLATON software, ${ }^{11}$ but their contributions were included in computing the overall formula and crystal density. The molecular structure and a view highlighting the coordination geometry are depicted in Figs. S6 and S7, respectively.

Physical Measurements. IR spectra were recorded on a Thermo Nicolet Avatar 360 spectrometer with OMNIC software. Mass spectra were recorded on an Agilent 1100 series LC/MSD electrospray MS. Benchtop UV-vis experiments were performed on a Hewlett-Packard 8453 diode array spectrophotometer. Stopped-flow kinetics measurements were performed on a Hi-Tech SF-61 stopped-flow spectrophotometer equipped with a diode array detector for recording absorption spectra of the reaction mixture. The kinetics data was collected and analyzed using Kinesis 3 program (HiTech). Conductivity measurements were performed with a Fisher Scientific portable conductivity meter (Model 09-326).

Kinetic Studies. The mechanism of the reaction between 2 and $\mathrm{Fe}(\mathrm{OTf})_{2} \cdot 2 \mathrm{MeCN}$ was studied by stopped-flow spectroscopy. The reaction temperature was maintained with a thermo-regulated cold $\mathrm{N}_{2}$ steam and a thermostat attached to the stopped-flow apparatus. The spectral changes upon mixing 2 with $\mathrm{Fe}(\mathrm{OTf})_{2} \cdot 2 \mathrm{MeCN}$ demonstrated the formation of the diiron(II) complex 3 (Fig. S8). The absorbance traces for the substitution reaction $(\lambda=570 \mathrm{~nm})$ fit well by a single exponential equation $\left[\mathrm{A}=\Delta \mathrm{A}\left(1-\mathrm{e}^{-k_{\mathrm{obs}} \mathrm{t}}\right)+\mathrm{A}_{0}\right]$ 
with an observed rate constant $\left(k_{\mathrm{obs}}\right)$ of $21 \pm 2 \mathrm{~s}^{-1}$, which were independent of the concentration of $\mathrm{Fe}(\mathrm{OTf})_{2} \cdot 2 \mathrm{MeCN}\left[\mathrm{Fig}\right.$. S9, final concentration for $\mathrm{Fe}(\mathrm{OTf})_{2} \cdot 2 \mathrm{MeCN}: 0.5$ $\mathrm{mM}$ (solid line with closed circles, $2 \mathrm{mM}$ (dotted line) and $4 \mathrm{mM}$ (solid line)]. Based on the equations derived for two-step reversible reactions, ${ }^{12}$ the observed rate constant following the formation of compound $\mathbf{3}$ in Scheme 3 should be the following:

$$
k_{\mathrm{obs}}=k_{1} \frac{k_{2}\left[\mathrm{Fe}(\mathrm{OTf})_{2} \cdot 2 \mathrm{MeCN}\right]}{k_{2}\left[\mathrm{Fe}(\mathrm{OTf})_{2} \cdot 2 \mathrm{MeCN}\right]+k_{-1}{ }^{\prime}}
$$

In this expression, $\left[\mathrm{Fe}(\mathrm{OTf})_{2} \cdot 2 \mathrm{MeCN}\right]$ is a constant because it is significantly higher than the [2]. The value of $k_{-1}{ }^{\prime}$ represents the reverse rate constant for the first equilibrium and equals $k_{-1}$ multiplied by $\left[\mathrm{Na}^{+}\right]$, if the latter is in excess compared with [2]. However, it is a variable in our experiment, because $\left[\mathrm{Na}^{+}\right]<[2]$. Nevertheless, the experimental observation that $k_{\mathrm{obs}}$ is independent of [Fe(OTf $\left.)_{2} \cdot 2 \mathrm{MeCN}\right]$ clearly demonstrates that $k_{2}\left[\mathrm{Fe}(\mathrm{OTf})_{2} \cdot 2 \mathrm{MeCN}\right]>>k_{-1}{ }^{\prime}$ and hence $k_{\mathrm{obs}} \approx k_{1}$.

The same reaction was also studied at various temperatures ranging from $-10{ }^{\circ} \mathrm{C}$ to $30{ }^{\circ} \mathrm{C}$ (Table 2). The values of $\ln \left(k_{\mathrm{obs}} / \mathrm{T}\right)$ were plotted against the reciprocal of reaction temperature (1/T) (Fig. S10). The enthalpy $\left(\Delta \mathrm{H}^{*}\right)$ and entropy $\left(\Delta \mathrm{S}^{\ddagger}\right)$ for the activation to the transition state were calculated based on the plot.

Conductivity Measurements. The conductivity meter was calibrated with a 3.42 $\mathrm{mM}$ aqueous solution of $\mathrm{NaCl}$ with a known specific conductance of $416 \mu \mathrm{S} \cdot \mathrm{cm}^{-1}$. The specific conductivities of $0.5-5 \mathrm{mM}$ acetonitrile solutions of $\mathbf{2}$ and $\mathbf{3}$ were measured at ambient temperature $\left(\sim 24{ }^{\circ} \mathrm{C}\right)$ inside the glovebox under a $\mathrm{N}_{2}$ atmosphere with stirring. Molar conductivities $\left(\Lambda_{\mathrm{M}}\right)$ were calculated from these values (Tables S3 and S4). For 2, $\Lambda_{\mathrm{M}}$ values were plotted against 2$]^{1 / 2}$ (Fig. S11). The molar conductivity at infinite 
dilution $\left(\Lambda_{0}=108 \mathrm{~S} \cdot \mathrm{cm}^{2} \cdot \mathrm{mol}^{-1}\right)$ was determined by extrapolating the plot to zero concentration. The dissociation constant $\mathrm{K}\left(\mathrm{k}_{1} / \mathrm{k}_{-1}\right)$ for 2 was calculated to be $2.3 \pm 0.4 \times 10^{-6} \mathrm{M}$ by using average $\mathrm{K}_{\mathrm{c}}$ values obtained from the Ostwald dilution law for a weak electrolyte, where $\mathrm{K}_{\mathrm{c}}=\left(\Lambda_{\mathrm{M}}\right)^{2} \mathrm{C} /\left(\Lambda_{0}\left(\Lambda_{0}-\Lambda_{\mathrm{M}}\right)\right.$.

\section{References}

${ }^{1}$ Pangborn, A. B.; Giardello, M. A.; Grubbs, R. H.; Rosen, R. K.; Timmers, F. J. Organometallics 1996, 15, 1518-1520.

${ }^{2}$ Kodanko, J. J.; Morys, A. J.; Lippard, S. J., Org. Lett., submitted for publication.

${ }^{3}$ Kawada, Y.; Iwamura, H.; J. Org. Chem. 1981, 46, 3357-3359.

${ }^{4}$ Farrell, J. R.; Stiles, D.; Bu, W.; Lippard, S. J. Tetrahedron, 2003, 59, 2463-2470.

${ }^{5}$ The identity of NaOTf was confirmed by IR spectroscopy. See Vardhan, H. B.; Bach, R. D. J. Org. Chem. 1992, 57, 4948-4954.

${ }^{6}$ SMART v5.626: Software for the CCD Detector System; Bruker AXS: Madison, WI, 2000.

${ }^{7}$ Kuzelka, J.; Mukhopadhyay, S.; Spingler, B.; Lippard, S. J. Inorg. Chem. 2004, 43, 1751-1761.

${ }^{8}$ Sheldrick, G. M. SHELXTL97-2: Program for Refinement of Crystal Structures; University of Göttingen: Göttingen, Germany, 1997.

${ }^{9}$ SHELXTL v5.10: Program Library for Structure Solution and Molecular Graphics; Bruker AXS: Madison, WI, 1998.

${ }^{10}$ Sheldrick, G. M. SADABS: Area-Detector Absorption Correction; University of Göttingen: Göttingen, Germany, 1996. 
${ }^{11}$ Spek, A. L. PLATON, A Multipurpose Crystallographic Tool; Utrecht University:

Utrecht, The Netherlands, 1998.

${ }^{12}$ Strickland, S.; Palmer, G.; Massey, V. J. Biol. Chem. 1975, 250, 4048-4052. 
Table S1. Summary of X-ray Crystallographic Data.

\begin{tabular}{|c|c|c|}
\hline & $\begin{array}{l}2 \text { (Structure A) } \\
{\left[\mathrm{NaFe}\left(\mathrm{PIC}_{2} \mathrm{DET}\right)(\mu-\right.} \\
\left.\left.\mathrm{O}_{2} \mathrm{CTrp}\right)_{3}\right] \cdot 2 \mathrm{CH}_{2} \mathrm{Cl}_{2} \cdot 0.5 \mathrm{Et}_{2} \mathrm{O}\end{array}$ & $\begin{array}{l}2 \text { (Structure B) } \\
{\left[\mathrm{NaFe}\left(\mathrm{PIC}_{2} \mathrm{DET}\right)(\mu-\right.} \\
\left.\left.\mathrm{O}_{2} \mathrm{CTrp}\right)_{3}\right] \cdot 4 \mathrm{CH}_{2} \mathrm{Cl}_{2}\end{array}$ \\
\hline Formula & $\mathrm{C}_{105} \mathrm{H}_{74} \mathrm{Cl}_{4} \mathrm{Fe} \mathrm{N} \mathrm{N}_{2} \mathrm{Na} \mathrm{O}_{10.50}$ & $\mathrm{C}_{105} \mathrm{H}_{71} \mathrm{Cl}_{8} \mathrm{Fe} \mathrm{N} \mathrm{N}_{2} \mathrm{Na} \mathrm{O}_{10}$ \\
\hline Formula Weight & 1752.30 & 1883.08 \\
\hline Space Group & $P \overline{1}$ & $P \overline{1}$ \\
\hline$a, \AA$ & $16.497(3)$ & $16.5031(7)$ \\
\hline$b, \AA$ & $17.251(4)$ & $16.7301(7)$ \\
\hline$c, \AA$ & $17.531(4)$ & $17.3222(8)$ \\
\hline$\alpha, \operatorname{deg}$ & $100.58(3)$ & $76.098(1)$ \\
\hline$\beta, \operatorname{deg}$ & $114.69(3)$ & $72.453(1)$ \\
\hline$\gamma, \operatorname{deg}$ & $100.46(3)$ & $76.436(1)$ \\
\hline $\mathrm{V}, \AA^{3}$ & $4266(2)$ & $4352(2)$ \\
\hline $\mathrm{Z}$ & 2 & 2 \\
\hline$\rho_{\text {calc }}, \mathrm{g} / \mathrm{cm}^{3}$ & 1.364 & 1.435 \\
\hline $\mathrm{T},{ }^{\circ} \mathrm{C}$ & $173(2) \mathrm{K}$ & 173(2) K \\
\hline$\mu(\mathrm{Mo} \mathrm{K} \alpha), \mathrm{mm}^{-1}$ & 0.373 & 0.489 \\
\hline$\theta$ limits, deg & $1.42-25.00$ & $1.70-25.00$ \\
\hline total no. of data & 31760 & 31528 \\
\hline no. of unique data & 14916 & 15212 \\
\hline no. of params & 1135 & 1045 \\
\hline GOF on $\mathrm{F}^{2}$ & 1.021 & 1.085 \\
\hline $\mathrm{R}_{1}(\%)^{\mathrm{a}}$ & 7.69 & 7.27 \\
\hline $\mathrm{wR}_{2}(\%)^{\mathrm{b}}$ & 20.98 & 20.57 \\
\hline $\max , \min$ peaks, $\mathrm{e} / \AA^{3}$ & $1.293,-1.099$ & $1.434,-0.747$ \\
\hline
\end{tabular}


Table S1, cont'd. Summary of X-ray Crystallographic Data, cont'd.

\begin{tabular}{|c|c|c|c|c|c|c|}
\hline \multirow{5}{*}{$\begin{array}{l} \\
\text { Selected } \\
\text { Interatomic } \\
\text { Distances, (̊) } \\
\text { and Angles, } \\
\text { (Deg) }\end{array}$} & \multicolumn{3}{|c|}{$\begin{array}{l}2 \text { (Structure A) } \\
{\left[\mathrm{NaFe}\left(\mathrm{PIC}_{2} \mathrm{DET}\right)\left(\mu-\mathrm{O}_{2} \mathrm{CTrp}\right)_{3}\right]} \\
\cdot 2 \mathrm{CH}_{2} \mathrm{Cl}_{2} \cdot 0.5 \mathrm{Et}_{2} \mathrm{O}\end{array}$} & \multicolumn{3}{|c|}{$\begin{array}{l}2 \text { (Structure B) } \\
{\left[\mathrm{NaFe}\left(\mathrm{PIC}_{2} \mathrm{DET}\right)\left(\mu-\mathrm{O}_{2} \mathrm{CTrp}\right)_{3}\right]} \\
\cdot 4 \mathrm{CH}_{2} \mathrm{Cl}_{2}\end{array}$} \\
\hline & $\mathrm{Fe}(1)-\mathrm{Na}(1)$ & 3.18 & & $\mathrm{Fe}(1)-\mathrm{Na}(1)$ & \multicolumn{2}{|c|}{$3.160(2)$} \\
\hline & $\mathrm{Fe}(1)-\mathrm{N}(1)$ & 2.19 & & $\mathrm{Fe}(1)-\mathrm{N}(1)$ & \multicolumn{2}{|c|}{$2.202(3)$} \\
\hline & $\mathrm{Fe}(1)-\mathrm{O}(2)$ & 2.18 & & $\mathrm{Fe}(1)-\mathrm{O}(1)$ & \multicolumn{2}{|c|}{$2.363(2)$} \\
\hline & $\mathrm{Fe}(1)-\mathrm{O}(5)$ & 2.60 & & $\mathrm{Fe}(1)-\mathrm{O}(5)$ & \multicolumn{2}{|c|}{$2.290(3)$} \\
\hline & $\mathrm{Fe}(1)-\mathrm{O}(6)$ & 2.13 & & $\mathrm{Fe}(1)-\mathrm{O}(7)$ & \multicolumn{2}{|c|}{$2.058(2)$} \\
\hline & $\mathrm{Fe}(1)-\mathrm{O}(7)$ & 2.03 & & $\mathrm{Fe}(1)-\mathrm{O}(9)$ & \multicolumn{2}{|c|}{$2.363(3)$} \\
\hline & $\mathrm{Fe}(1)-\mathrm{O}(9)$ & 2.32 & & $\mathrm{Fe}(1)-\mathrm{O}(10)$ & \multicolumn{2}{|c|}{$2.161(3)$} \\
\hline & $\mathrm{Fe}(1)-\mathrm{O}(10)$ & 2.18 & & $\mathrm{Na}(1)-\mathrm{N}(2)$ & \multicolumn{2}{|c|}{$2.508(3)$} \\
\hline & $\mathrm{Na}(1)-\mathrm{N}(2)$ & 2.50 & & $\mathrm{Na}(1)-\mathrm{O}(3)$ & \multicolumn{2}{|c|}{$2.382(3)$} \\
\hline & $\mathrm{Na}(1)-\mathrm{O}(3)$ & 2.34 & & $\mathrm{Na}(1)-\mathrm{O}(6)$ & \multicolumn{2}{|c|}{$2.143(3)$} \\
\hline & $\mathrm{Na}(1)-\mathrm{O}(6)$ & 2.30 & & $\mathrm{Na}(1)-\mathrm{O}(8)$ & \multicolumn{2}{|c|}{$2.232(3)$} \\
\hline & $\mathrm{Na}(1)-\mathrm{O}(8)$ & 2.28 & & $\mathrm{Na}(1)-\mathrm{O}(9)$ & \multicolumn{2}{|c|}{$2.287(3)$} \\
\hline & $\mathrm{Na}(1)-\mathrm{O}(9)$ & 2.28 & & $\mathrm{O}(1)-\mathrm{C}(1)$ & \multicolumn{2}{|c|}{$1.472(4)$} \\
\hline & $\mathrm{O}(1)-\mathrm{C}(2)$ & 1.31 & & $\mathrm{O}(1)-\mathrm{C}(2)$ & \multicolumn{2}{|c|}{$1.326(4)$} \\
\hline & $\mathrm{O}(1)-\mathrm{C}(1)$ & 1.45 & & $\mathrm{O}(2)-\mathrm{C}(2)$ & \multicolumn{2}{|c|}{$1.202(4)$} \\
\hline & $\mathrm{O}(2)-\mathrm{C}(2)$ & 1.20 & & $\mathrm{O}(3)-\mathrm{C}(37)$ & \multicolumn{2}{|c|}{$1.211(5)$} \\
\hline & $\mathrm{O}(3)-\mathrm{C}(37)$ & 1.19 & & $\mathrm{O}(4)-\mathrm{C}(37)$ & \multicolumn{2}{|c|}{$1.303(5)$} \\
\hline & $\mathrm{O}(4)-\mathrm{C}(37)$ & 1.32 & & $\mathrm{O}(4)-\mathrm{C}(38)$ & \multicolumn{2}{|c|}{$1.466(4)$} \\
\hline & $\mathrm{O}(4)-\mathrm{C}(38)$ & 1.44 & & & & \\
\hline & \multicolumn{2}{|c|}{$\mathrm{O}(7)-\mathrm{Fe}(1)-\mathrm{O}(6)$} & $93.7(1)$ & $\mathrm{O}(7)-\mathrm{Fe}(1)-\mathrm{O}$ & & $90.9(1)$ \\
\hline & \multicolumn{2}{|c|}{$\mathrm{O}(7)-\mathrm{Fe}(1)-\mathrm{O}(10)$} & $97.7(1)$ & \multicolumn{2}{|c|}{$\mathrm{O}(7)-\mathrm{Fe}(1)-\mathrm{O}(10)$} & $88.6(1)$ \\
\hline & \multicolumn{2}{|c|}{$\mathrm{O}(6)-\mathrm{Fe}(1)-\mathrm{O}(10)$} & $139.0(1)$ & \multicolumn{2}{|c|}{$\mathrm{O}(10)-\mathrm{Fe}(1)-\mathrm{O}(5)$} & $173.3(1)$ \\
\hline & \multicolumn{2}{|c|}{$\mathrm{O}(7)-\mathrm{Fe}(1)-\mathrm{O}(2)$} & $85.5(1)$ & \multicolumn{2}{|c|}{$\mathrm{O}(7)-\mathrm{Fe}(1)-\mathrm{O}(1)$} & $82.8(1)$ \\
\hline & \multicolumn{2}{|c|}{$\mathrm{O}(6)-\mathrm{Fe}(1)-\mathrm{O}(2)$} & $131.9(1)$ & \multicolumn{2}{|c|}{$\mathrm{O}(5)-\mathrm{Fe}(1)-\mathrm{O}(1)$} & $83.4(1)$ \\
\hline & $\mathrm{O}(10)-\mathrm{Fe}(1)$ & $\mathrm{O}(2)$ & $88.3(1)$ & $\mathrm{O}(10)-\mathrm{Fe}(1)-$ & & $89.9(1)$ \\
\hline & $\mathrm{O}(7)-\mathrm{Fe}(1)-\mathrm{I}$ & $(1)$ & $159.3(1)$ & $\mathrm{O}(7)-\mathrm{Fe}(1)-\mathrm{N}$ & & $153.8(1)$ \\
\hline & $\mathrm{O}(6)-\mathrm{Fe}(1)-\mathrm{I}$ & (1) & $94.3(1)$ & $\mathrm{O}(5)-\mathrm{Fe}(1)-\mathrm{N}$ & & $84.8(1)$ \\
\hline & $\mathrm{O}(10)-\mathrm{Fe}(1)$ & $\mathrm{N}(1)$ & $88.7(1)$ & $\mathrm{O}(10)-\mathrm{Fe}(1)-\mathrm{I}$ & & $92.6(1)$ \\
\hline & $\mathrm{O}(2)-\mathrm{Fe}(1)-\mathrm{I}$ & (1) & $75.0(1)$ & $\mathrm{O}(1)-\mathrm{Fe}(1)-\mathrm{N}$ & & $71.05(9)$ \\
\hline & $\mathrm{O}(6)-\mathrm{Fe}(1)-\mathrm{I}$ & $\mathrm{a}(1)$ & $46.5(1)$ & $\mathrm{O}(5)-\mathrm{Fe}(1)-\mathrm{N}$ & & $94.47(8)$ \\
\hline & $\mathrm{O}(8)-\mathrm{Na}(1)-$ & $(9)$ & $97.7(1)$ & $\mathrm{O}(8)-\mathrm{Na}(1)-\mathrm{C}$ & & $92.8(1)$ \\
\hline
\end{tabular}




$\mathrm{O}(8)-\mathrm{Na}(1)-\mathrm{O}(6)$
$\mathrm{O}(9)-\mathrm{Na}(1)-\mathrm{O}(6)$
$\mathrm{O}(8)-\mathrm{Na}(1)-\mathrm{O}(3)$
$\mathrm{O}(9)-\mathrm{Na}(1)-\mathrm{O}(3)$
$\mathrm{O}(6)-\mathrm{Na}(1)-\mathrm{O}(3)$
$\mathrm{O}(8)-\mathrm{Na}(1)-\mathrm{N}(2)$
$\mathrm{O}(9)-\mathrm{Na}(1)-\mathrm{N}(2)$
$\mathrm{O}(6)-\mathrm{Na}(1)-\mathrm{N}(2)$
$\mathrm{O}(3)-\mathrm{Na}(1)-\mathrm{N}(2)$

$\mathrm{O}(8)-\mathrm{Na}(1)-\mathrm{O}(6)$

88.6(1) $\mathrm{O}(8)-\mathrm{Na}(1)-\mathrm{O}(6)$

78.6(1) $\mathrm{O}(9)-\mathrm{Na}(1)-\mathrm{O}(6)$

101.1(1) O(8)-Na(1)-O(3)

142.6(2) $\mathrm{O}(9)-\mathrm{Na}(1)-\mathrm{O}(3)$

133.7(1) $\mathrm{O}(6)-\mathrm{Na}(1)-\mathrm{O}(3)$

167.7(2) $\mathrm{O}(8)-\mathrm{Na}(1)-\mathrm{N}(2)$

94.5(1) O(9)-Na(1)-N(2)

95.5(1) O(6)-Na(1)-N(2)

67.8(1) O(3)-Na(1)-N(2)
89.9(1)

$85.8(1)$

103.3(1)

154.8(1)

113.2(1)

168.3(1)

98.4(1)

87.4(1)

$67.6(1)$ 
Table S2. Temperature dependence of the observed rate constant $\left(k_{\mathrm{obs}}\right)$ for the reaction of 2 with $\mathrm{Fe}(\mathrm{OTf})_{2} \cdot 2 \mathrm{MeCN}$ in $\mathrm{MeCN}\left([2]=0.5 \mathrm{mM},\left[\mathrm{Fe}(\mathrm{OTf})_{2} \cdot 2 \mathrm{MeCN}\right]=4.0 \mathrm{mM}\right.$ after mixing). Rate constants were obtained by averaging values from quadruplicate reactions at each temperature. Error values include a correction for temperature instability.

\begin{tabular}{ll}
\hline $\mathrm{T}(\mathrm{K})$ & $k_{\mathrm{obs}}\left(\mathrm{s}^{-1}\right)$ \\
\hline 263.13 & $1.0 \pm 0.2$ \\
268.13 & $1.6 \pm 0.2$ \\
273.13 & $2.4 \pm 0.1$ \\
278.12 & $3.3 \pm 0.1$ \\
283.13 & $4.5 \pm 0.2$ \\
288.13 & $9.3 \pm 1.4$ \\
293.13 & $16.4 \pm 0.1$ \\
298.13 & $24.3 \pm 1.6$ \\
303.13 & $41.0 \pm 1.4$ \\
\hline
\end{tabular}


Table S3. Molar conductivities for 2 in $\mathrm{MeCN}$ at $24^{\circ} \mathrm{C}$.

\begin{tabular}{ll}
\hline$[2]$ in $\mathrm{MeCN}(\mathrm{mM})$ & $\Lambda_{\mathrm{M}}\left(\mathrm{S} \cdot \mathrm{cm}^{2} \cdot \mathrm{mol}^{-1}\right)$ \\
\hline 5.0 & 56.0 \\
2.5 & 60.4 \\
1.0 & 79.8 \\
0.5 & 91.8
\end{tabular}

Table S4. Molar conductivities for 3 in $\mathrm{MeCN}$ at $24^{\circ} \mathrm{C}$.

\begin{tabular}{ll}
\hline$[2]$ in $\mathrm{MeCN}(\mathrm{mM})$ & $\Lambda_{\mathrm{M}}\left(\mathrm{S} \cdot \mathrm{cm}^{2} \cdot \mathrm{mol}^{-1}\right)$ \\
\hline 5.0 & 120.2 \\
2.5 & 126.8 \\
1.0 & 141.1 \\
0.5 & 155.6
\end{tabular}


Figure S1. UV-vis spectra of 2, $4 \mathrm{mM}$ in $\mathrm{CH}_{2} \mathrm{Cl}_{2}$ and the product of the biphasic reaction of $4 \mathrm{mM} 2$ in $\mathrm{CH}_{2} \mathrm{Cl}_{2}$ treated with 5 equiv of solid $\mathrm{Fe}(\mathrm{OTf})_{2} \cdot 2 \mathrm{MeCN}$ and filtered prior to recording the spectrum.

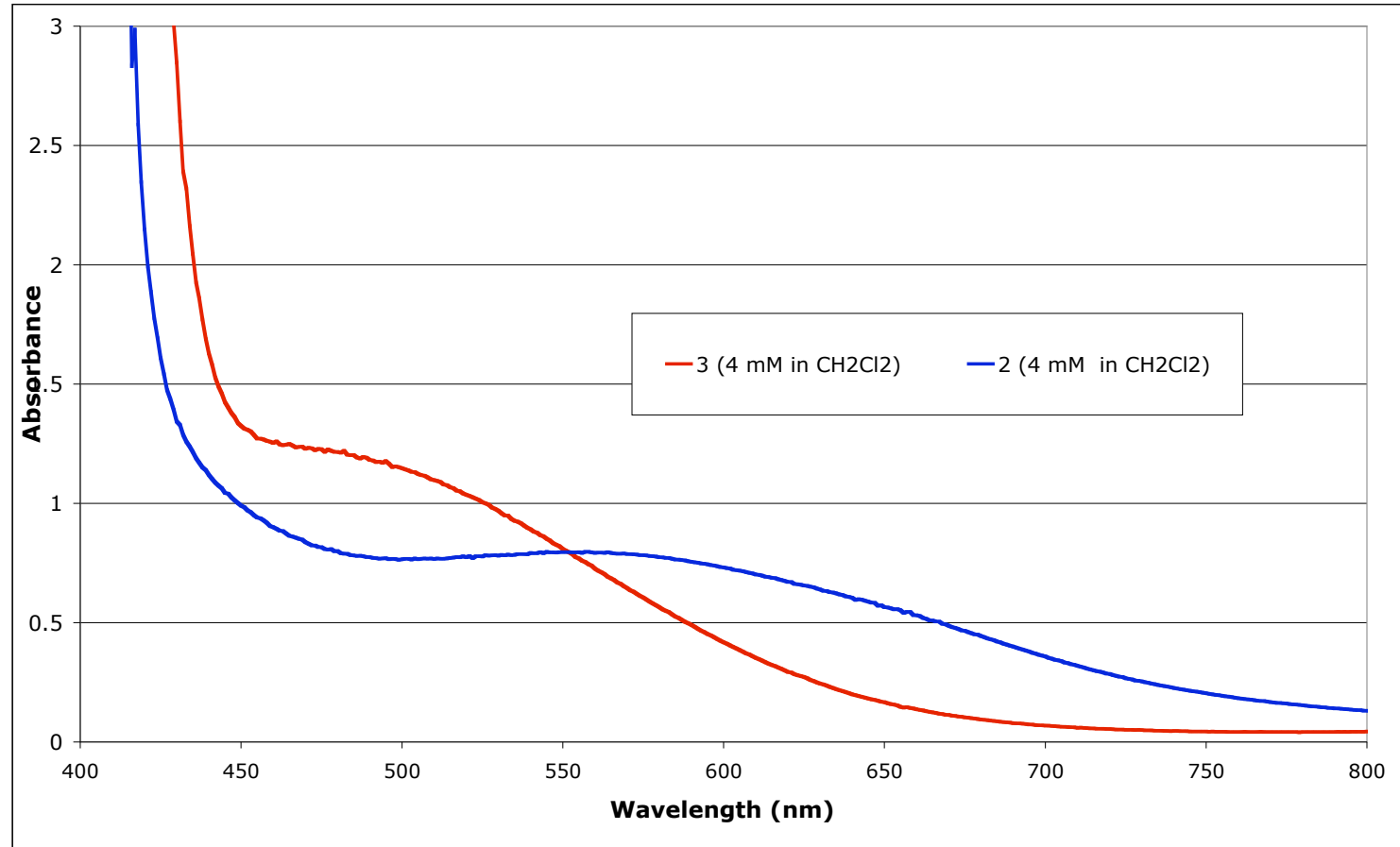


Figure S2. UV-vis spectra of $4 \mathrm{mM}$ solutions of $\mathbf{2}$ and $\mathbf{3}$ in $\mathrm{MeCN}$.

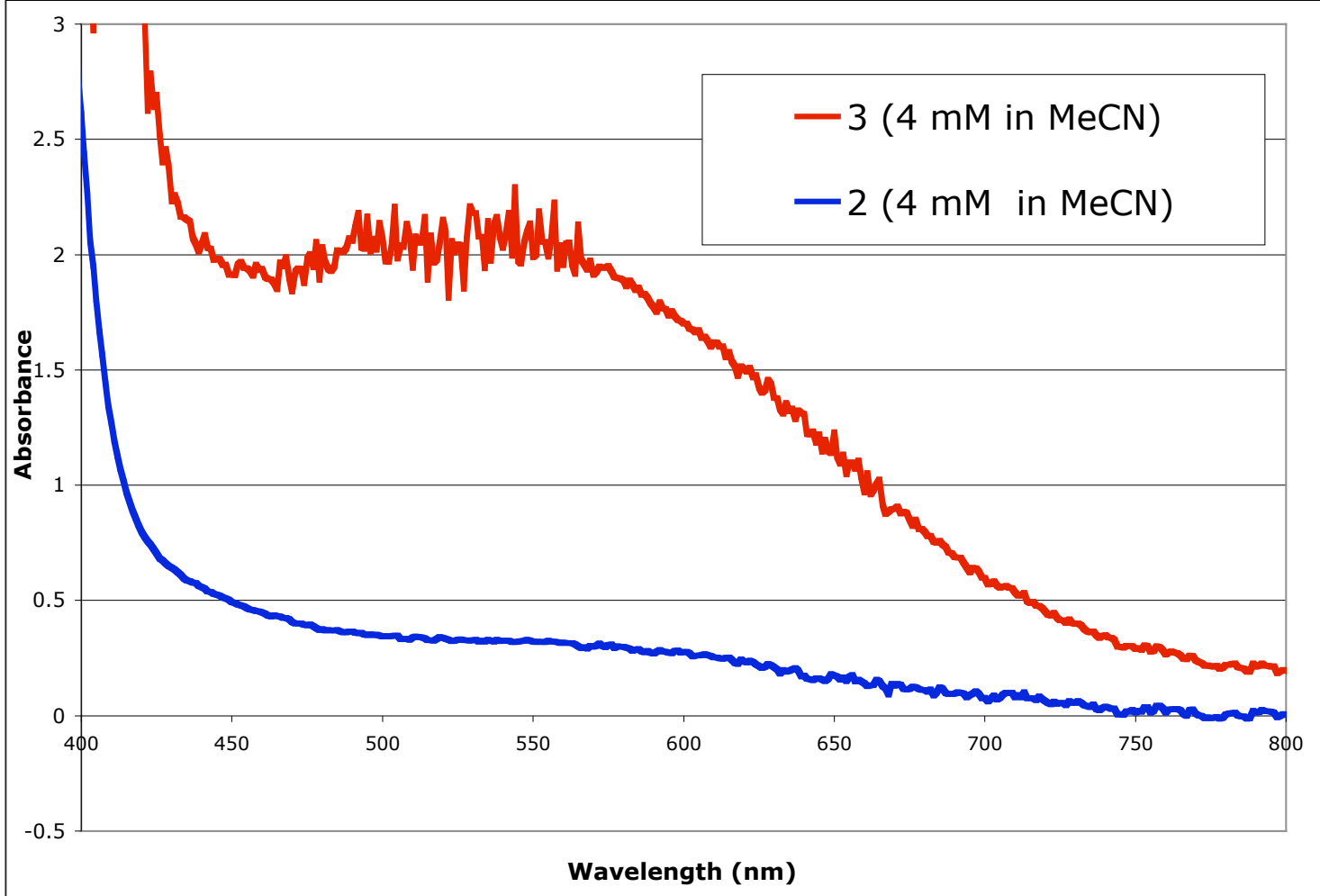


Figure S3. UV-vis spectra from the titration of 2 with 4 equiv of $\mathrm{Fe}(\mathrm{OTf})_{2} \cdot 2 \mathrm{MeCN}$ in $\operatorname{MeCN}([2]=1 \mathrm{mM})$.

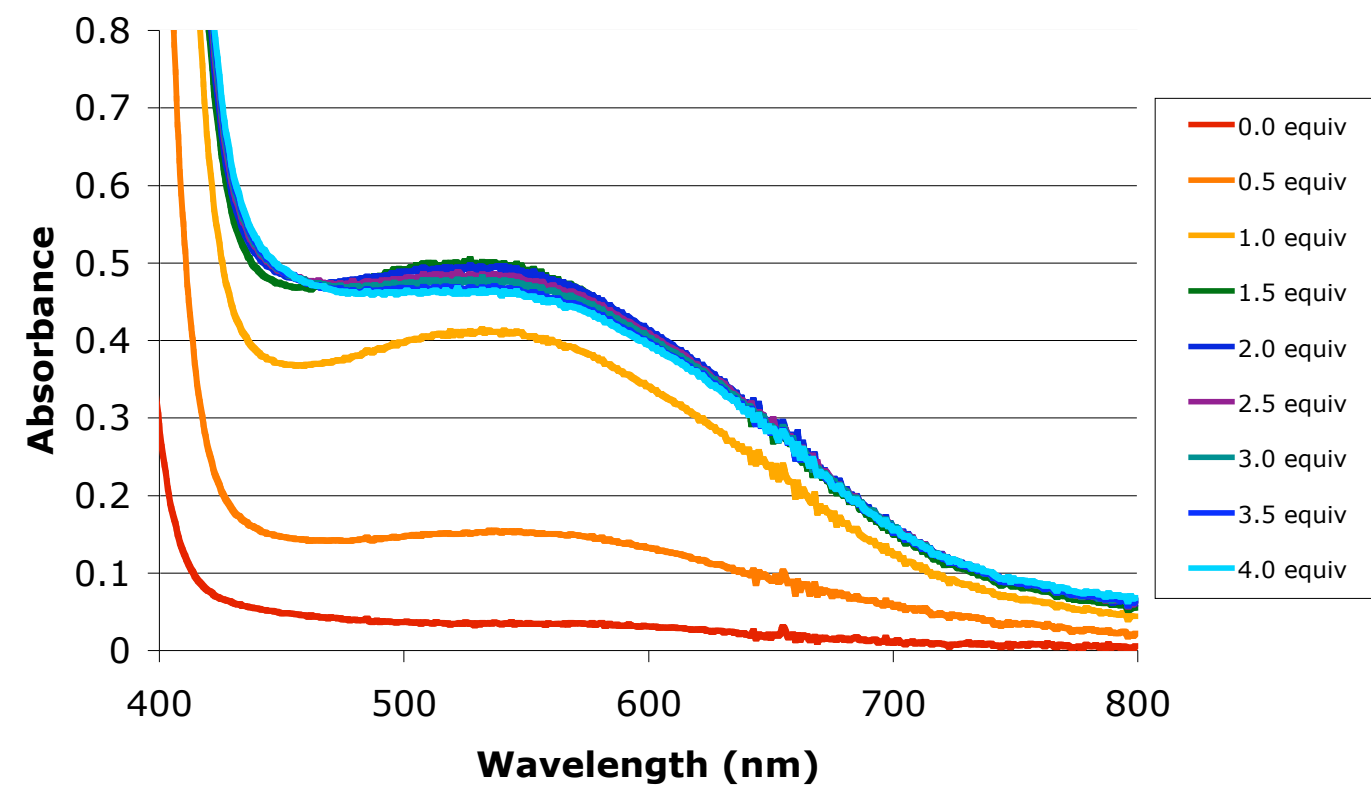


Figure S4. $\left[\mathrm{NaFe}\left(\mathrm{PIC}_{2} \mathrm{DET}\right)\left(\mu-\mathrm{O}_{2} \mathrm{CTrp}\right)_{3}\right]$ (2) full Structure A showing 30\% probability thermal ellipsoids for all non-hydrogen atoms.

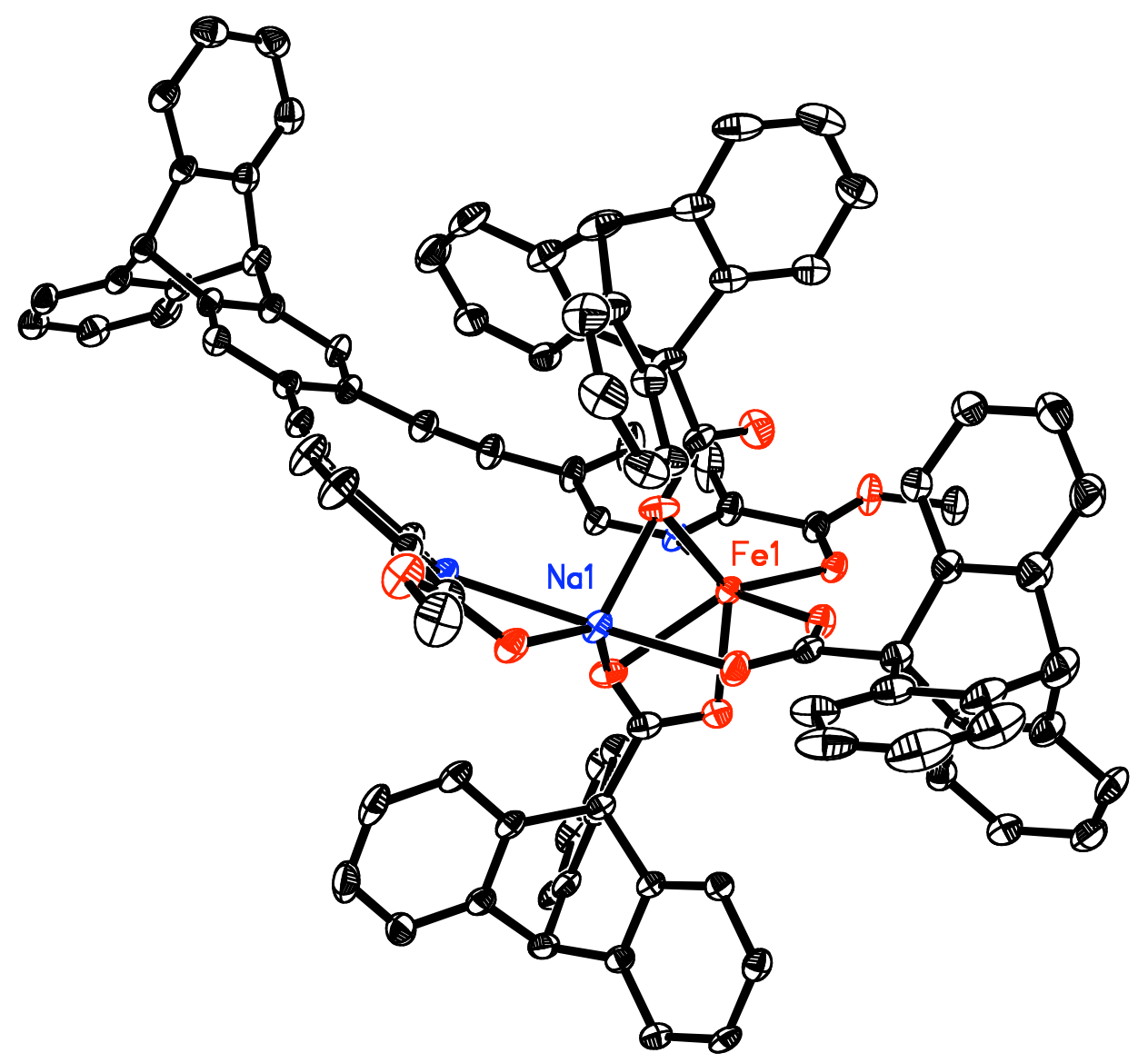


Figure S5. $\left[\mathrm{NaFe}\left(\mathrm{PIC}_{2} \mathrm{DET}\right)\left(\mu-\mathrm{O}_{2} \mathrm{CTrp}\right)_{3}\right]$ (2) abbreviated Structure A showing $30 \%$ probability thermal ellipsoids for all non-hydrogen atoms.

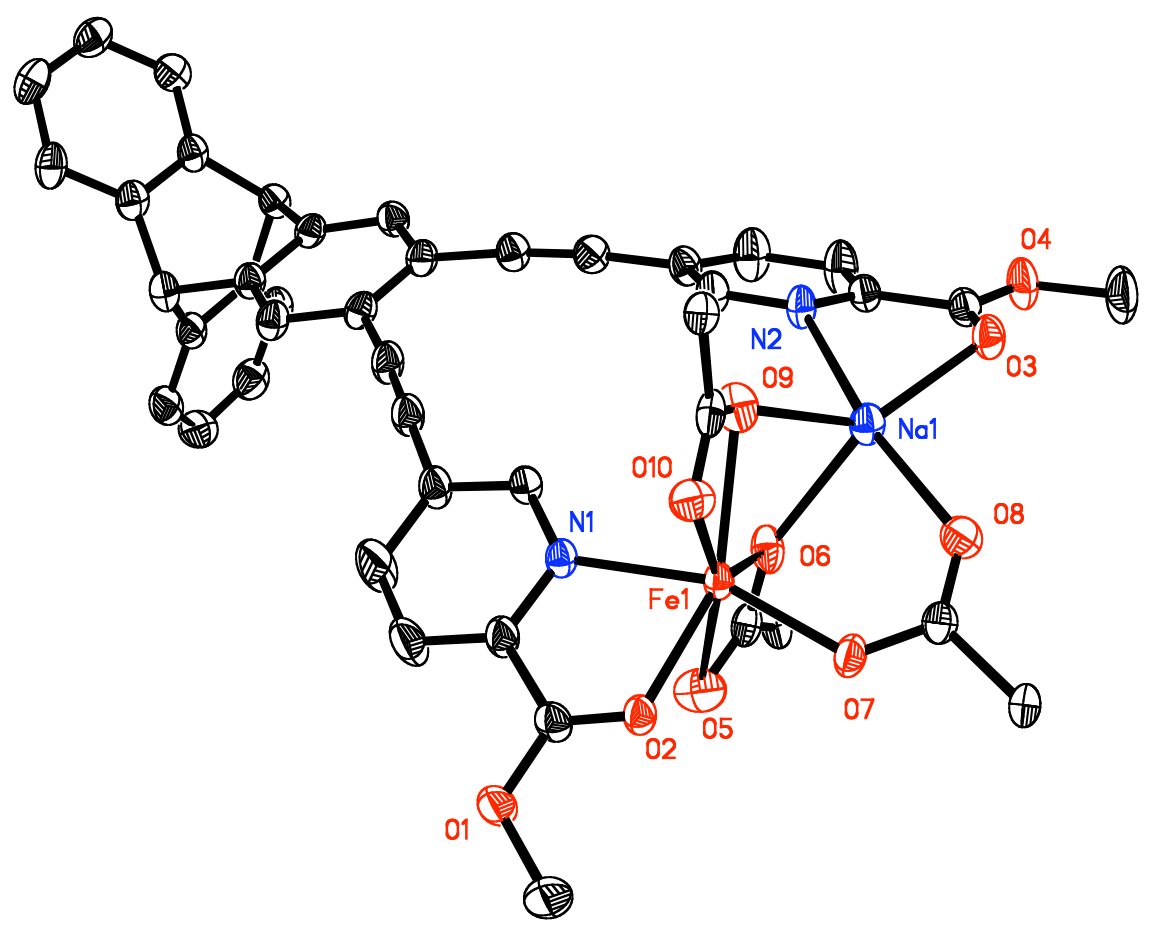


Figure S6. $\left[\mathrm{NaFe}\left(\mathrm{PIC}_{2} \mathrm{DET}\right)\left(\mu-\mathrm{O}_{2} \mathrm{CTrp}\right)_{3}\right]$ (2) full Structure B showing $30 \%$ probability thermal ellipsoids for all non-hydrogen atoms.

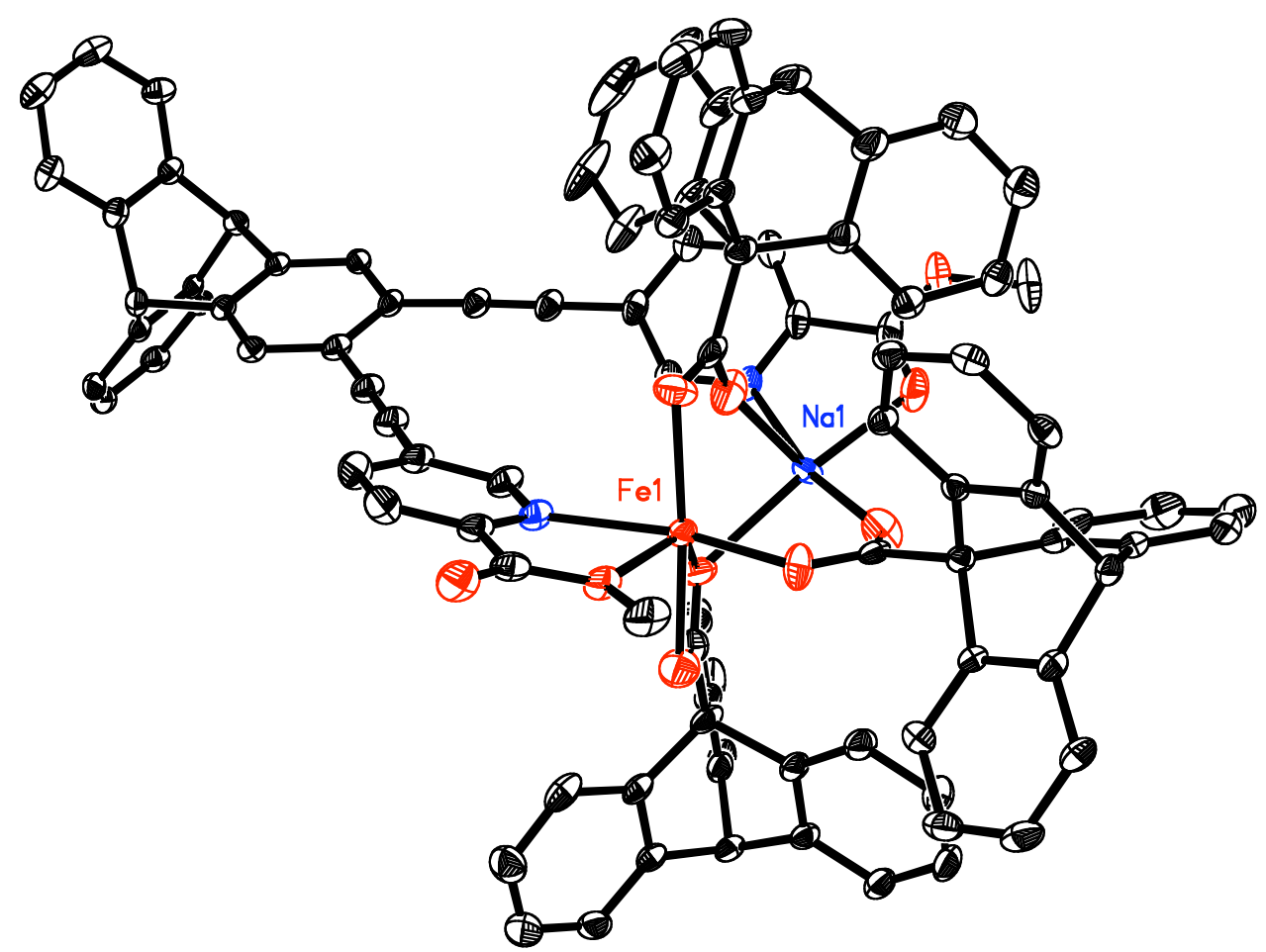


Figure S7. $\left[\mathrm{NaFe}\left(\mathrm{PIC}_{2} \mathrm{DET}\right)\left(\mu-\mathrm{O}_{2} \mathrm{CTrp}\right)_{3}\right]$ (2) abbreviated Structure B showing $30 \%$ probability thermal ellipsoids for all non-hydrogen atoms.

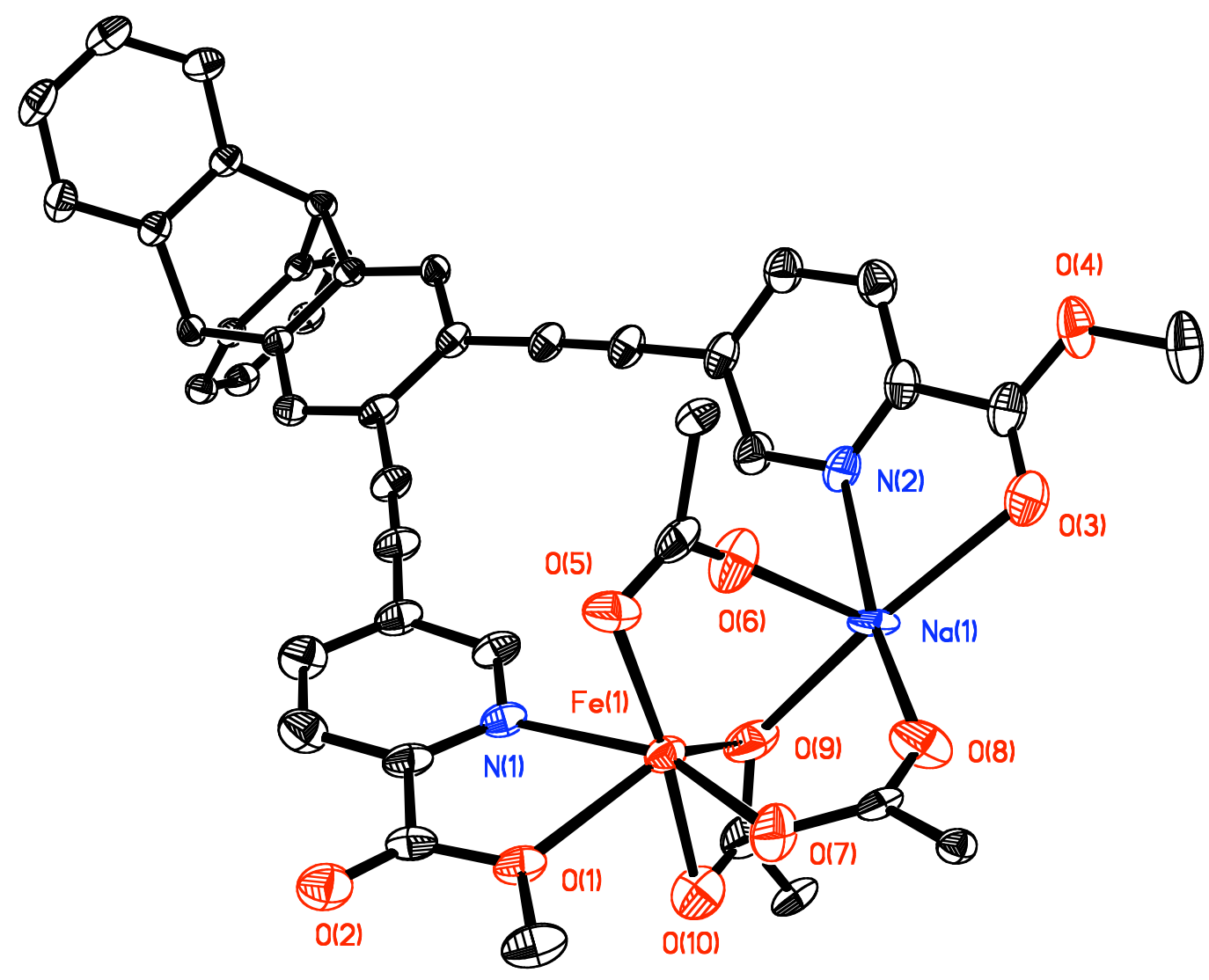


Figure S8. Spectral changes upon mixing 2 with $\mathrm{Fe}(\mathrm{OTf})_{2} \cdot 2 \mathrm{MeCN}$ in a diode array stopped-flow spectrometer. A total of 160 spectra were acquired between $2 \mathrm{~ms}$ and $5 \mathrm{sec}$ on a logarithmic time scale.

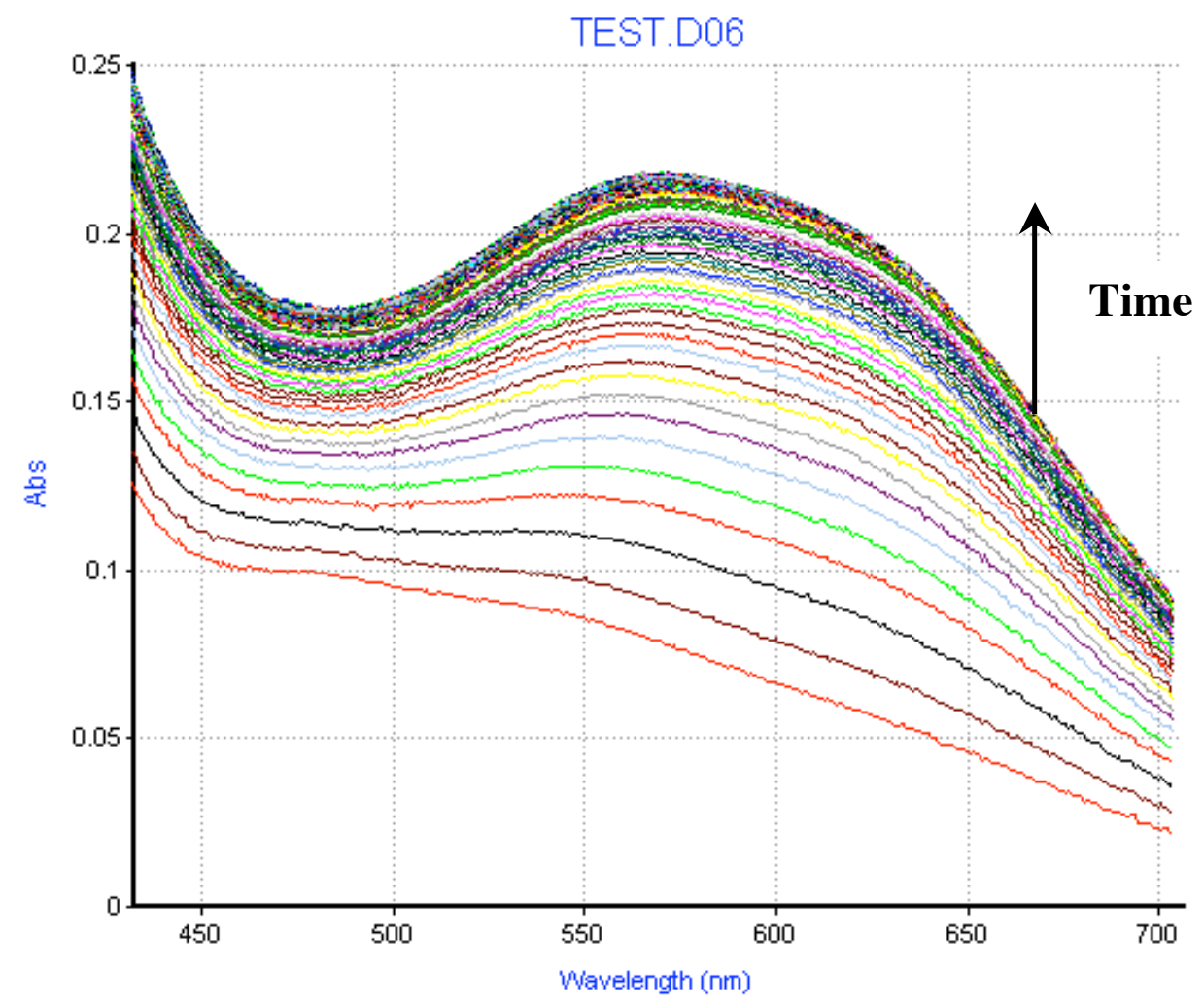


Figure S9. Traces from reaction of 2 and $\mathrm{Fe}(\mathrm{OTf})_{2} \cdot 2 \mathrm{MeCN}$ in $\mathrm{MeCN}$. The final concentrations of 2 is $0.5 \mathrm{mM}$ and for $\mathrm{Fe}(\mathrm{OTf})_{2} \cdot 2 \mathrm{MeCN}$ are $0.5 \mathrm{mM}$ (solid line with closed circles), $2 \mathrm{mM}$ (dotted line) and $4 \mathrm{mM}$ (solid line).

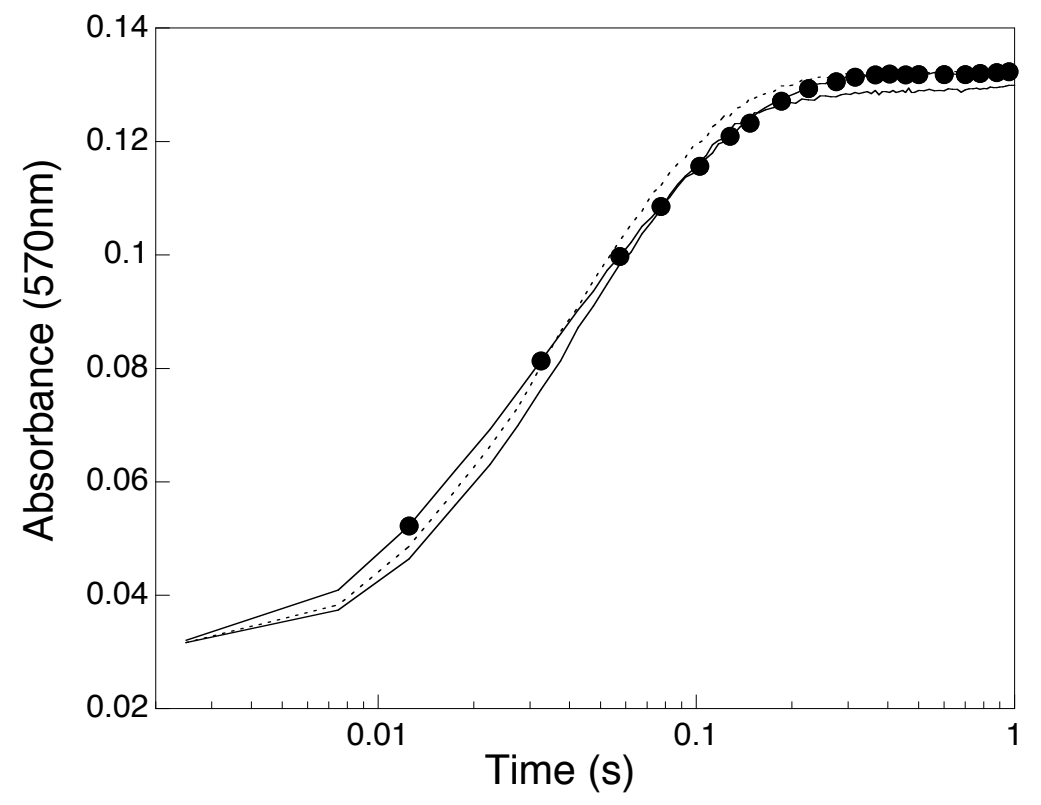


Figure S10. The temperature dependence of $k_{\mathrm{obs}}$ for reaction of $\mathbf{2}$ and $\mathrm{Fe}(\mathrm{OTf})_{2} \cdot 2 \mathrm{MeCN}$ in $\mathrm{MeCN}$

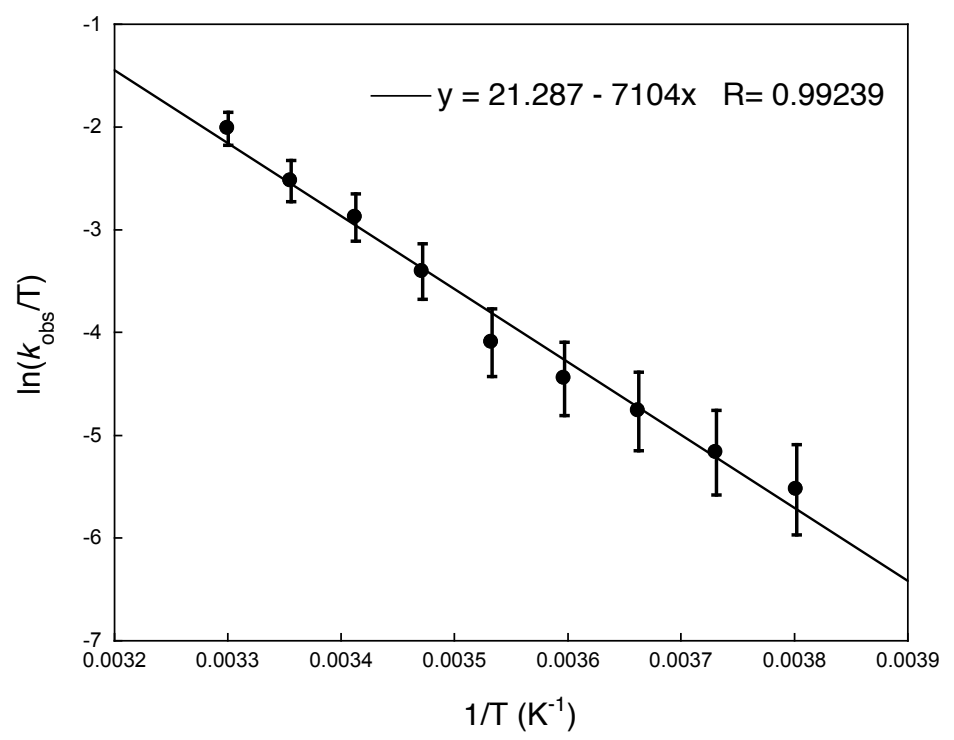

$$
\begin{aligned}
& \Delta \mathrm{H}^{\ddagger}=-(\text { slope }) \times \mathrm{R}=7.1 \times 10^{3} \times 8.314=59( \pm 6)\left(\mathrm{kJ} \mathrm{mol}^{-1}\right) \\
& \Delta \mathrm{S}^{\ddagger}=\Delta \mathrm{H}^{\ddagger} / \mathrm{T}+\mathrm{R} \times \ln \left(\mathrm{h} k_{\mathrm{r}} / \mathrm{\kappa kT}\right) \\
& \mathrm{R}=8.314 \mathrm{~J} \mathrm{~mol}^{-1} \mathrm{~K}^{-1}, \mathrm{~h}=6.63 \times 10^{-34} \mathrm{~J} \mathrm{~s}, \mathrm{k}=1.38 \times 10^{-23} \mathrm{~J} \mathrm{~K}^{-1}, \kappa=1
\end{aligned}
$$

\begin{tabular}{llc}
\hline $\mathrm{T}(\mathrm{K})$ & $k_{\mathrm{r}}=k_{\mathrm{obs}}\left(\mathrm{s}^{-1}\right)$ & $\left.\Delta \mathrm{S}^{\ddagger} \mathrm{J} \mathrm{mol}^{-1} \mathrm{~K}^{-1}\right)$ \\
\hline 263.13 & $1.0 \pm 0.2$ & -17 \\
268.13 & $1.6 \pm 0.2$ & -15 \\
273.13 & $2.4 \pm 0.1$ & -16 \\
278.12 & $3.3 \pm 0.1$ & -18 \\
283.13 & $4.5 \pm 0.2$ & -20 \\
288.13 & $9.3 \pm 1.4$ & -22 \\
293.13 & $16.4 \pm 0.1$ & -24 \\
298.13 & $24.3 \pm 1.6$ & -27 \\
303.13 & $41.0 \pm 1.4$ & -29 \\
\hline Average & & $-20 \pm 6$ \\
\hline
\end{tabular}


Figure S11. Plot of the molar conductivity of $2\left(\Lambda_{\mathrm{M}}\right)$ versus $[2]^{1 / 2}$ in $\mathrm{MeCN}$ at $24^{\circ} \mathrm{C}$.

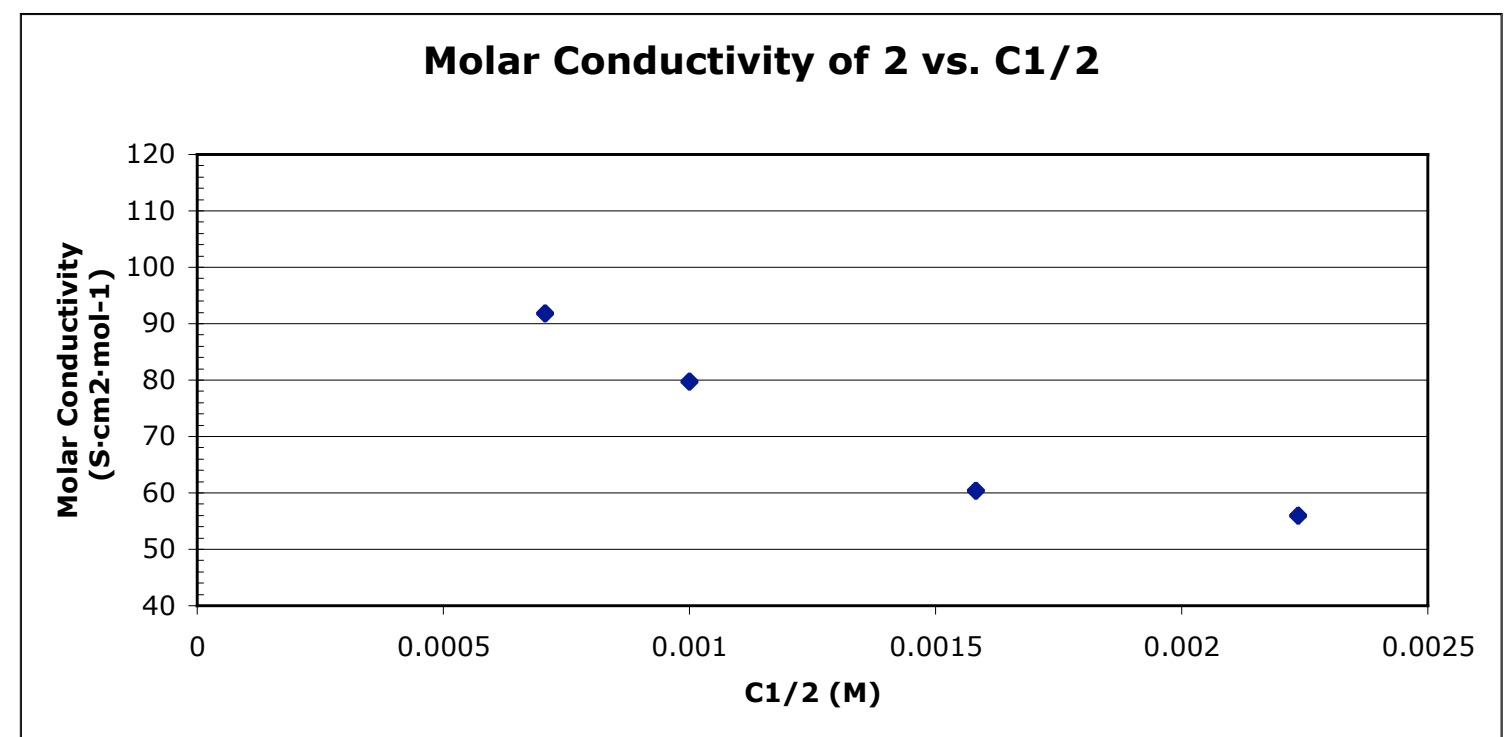

